\title{
When should principals acquire verifiable information?
}

Citation for published version (APA):

Feess, E., Schieble, M., \& Walzl, M. (2004). When should principals acquire verifiable information? METEOR, Maastricht University School of Business and Economics. METEOR Research Memorandum No. 047 https://doi.org/10.26481/umamet.2004047

Document status and date:

Published: 01/01/2004

DOI:

10.26481/umamet.2004047

Document Version:

Publisher's PDF, also known as Version of record

\section{Please check the document version of this publication:}

- A submitted manuscript is the version of the article upon submission and before peer-review. There can be important differences between the submitted version and the official published version of record.

People interested in the research are advised to contact the author for the final version of the publication, or visit the DOI to the publisher's website.

- The final author version and the galley proof are versions of the publication after peer review.

- The final published version features the final layout of the paper including the volume, issue and page numbers.

Link to publication

\footnotetext{
General rights rights.

- You may freely distribute the URL identifying the publication in the public portal. please follow below link for the End User Agreement:

www.umlib.nl/taverne-license

Take down policy

If you believe that this document breaches copyright please contact us at:

repository@maastrichtuniversity.nl

providing details and we will investigate your claim.
}

Copyright and moral rights for the publications made accessible in the public portal are retained by the authors and/or other copyright owners and it is a condition of accessing publications that users recognise and abide by the legal requirements associated with these

- Users may download and print one copy of any publication from the public portal for the purpose of private study or research.

- You may not further distribute the material or use it for any profit-making activity or commercial gain

If the publication is distributed under the terms of Article $25 \mathrm{fa}$ of the Dutch Copyright Act, indicated by the "Taverne" license above, 


\title{
When Should Principals Acquire Verifiable Information?*
}

\author{
Eberhard Feess ${ }^{\dagger}$ Michael Schieble ${ }^{\ddagger}$ and Markus Walzl ${ }^{\S}$
}

June 2, 2004

\begin{abstract}
We analyze a principal-agent model in which a principal has two possibilities to improve his knowledge about the quality of an investment project. First, he has access to an information technology that provides a verifiable, unbiased signal. Second, he can hire an agent who detects bad projects with some probability depending on his unobservable effort, and who reports his findings opportunistically. We analyze whether the principal should check the signal before or after he offers a contract. The first policy has the advantage that the agent's effort can be adjusted to the signal, whereas the second policy allows a reduction in the agent's rent. We show that checking the signal afterwards is always superior if the signal is sufficiently uninformative. Keywords: information revelation, hidden action, limited liability. JEL classification: D82, G21
\end{abstract}

\footnotetext{
*We are grateful to Sandro Gleave, Uli Hege, Ernst Maug, Gerd Muehlheusser and Martin Nell.

$\dagger^{\dagger}$ Department of Economics, Aachen University of Technology (RWTH), Templergraben 64, 52062 Aachen, Germany. Phone: 0049-241-8096155. email: feess@rwth-aachen.de.

¥Bain\&Company, Munich. email:Michael.Schieble@bain.com.

${ }^{\S}$ Department of Economics and METEOR, University of Maastricht, P.O. Box 616, NL-6200 MD Maastricht, The Netherlands. Phone: 0031-43-3883807. email: m.walzl@algec.unimaas.nl.
} 


\section{Introduction}

Motivation and main results We analyze a model in which a principal owns a project that can be either good or bad. The principal has two possibilities to improve his knowledge about the project's quality. On the one hand, he can use an information technology (IT) that is not subject to any kind of incentive problem. We assume that the technology provides a verifiable signal on the project's quality. On the other hand, the principal can hire an agent who can detect bad projects with some probability depending on his unobservable effort. After having detected a bad project or not, the agent reports his findings opportunistically to the principal. Hence, engaging the agent is not only subject to moral hazard, but also to a truth-telling (adverse selection) problem. Furthermore, the agent is assumed to have zero wealth, so that all payments from the principal to the agent need to be non-negative. The main question analyzed in our paper is whether the principal should commit himself to check the IT before or after the agent has reported his findings. The first policy will be called "disclosure policy $(D)$ ", since the outcome of the IT is known to both the principal and the agent before the principal offers a contract. In the second policy, labelled as "concealment policy $(C)$ ", the IT will be checked only after the agent has reported his findings to the principal. Of course, the advantage of policy $D$ is that the agent can adjust his effort to the principal's findings. On the other hand, policy $C$ allows the principal to reduce the agent's rent caused by the truthtelling problem by threatening to pay positive wages if and only if the agent's report and the signal's outcome coincide.

Our setting seems to fit for many practically relevant instances. Originally, the analysis has been motivated by a project concerning scoring systems for small enterprize loans. Loan officers who are supposed to improve and to reveal their own knowledge from relationship lending to their superiors often seem to duplicate the scoring system's result without working seriously, thus motivating the issue whether committing to run the scoring system (which provides verifiable information) only after the loan officer's assessment might be better. More generally, our framework fits whenever the principal has one source of information that is not or no longer subject to a moral hazard or truth-telling problem - for instance, a lawyer's client, a doctor's patient or an editor may already have an other report waiting sealed on his desk to be opened, and he can decide (and convey) when to open the report. In a similar way, an investor or a CEO may consider when to undertake an 
in-house database research complementary to the (out-sourced) work of a consulting agency.

In this contribution, we derive the optimal timing of information release depending on the quality of the principals IT, the project characteristics and the agent's effort-cost function that determines the magnitude of moral hazard and adverse selection effects.

The most surprising results under policy $D$ itself are that the principal may maximize profits by not using the IT at all, and that his profit may decrease in the number of good projects. These results hold although we assume that using the IT is costless, and are caused by the truth-telling problem. By contrast, the principal always checks the IT under policy $C$, and his profit strictly increases in the percentage of good projects.

When comparing the relative advantages of the two policies, our results are as follows. First, policy $D$ is superior if there is no truth-telling problem, but only a moral hazard problem (with limited liability). Second, policy $C$ is superior whenever the informativeness of a good signal is sufficiently low, or when the principal prefers not to check the signal under policy $D$. Third, policy $D$ is not necessarily superior if the signal becomes arbitrarily informative as the principal will refuse to hire the agent at all in this case.

Relation to the literature To the best of our knowledge, Demski and Sappington (DS 1987b) is the only paper that addresses the question of the optimal timing of information release in a principal-agent relationship. DS 1987b is an extension of Demski and Sappington (DS 1987a) that pioneers the literature on delegated expertise. In these models, the principal delegates both the information acquisition and a subsequent implementation decision (upon an investment, for instance) to the agent because communication is prohibitively costly. In DS 1987b, the agent's unobservable effort in acquiring information is binary, and the optimal (non-binary) implementation decision depends on both the agent's and the principal's information. Then, they provide examples for the optimality of the different timings of information release. However, the trade-off between rent payments and effort selection in the optimal contract which is the focus of our paper cannot be analyzed in their binary-effort model.

Furthermore, our analysis is related to the literature on informed principals developed by Maskin and Tirole (MT 1990), Maskin and Tirole (MT 1992) and Beaudry (1994) where the principal may signal his private information 
through the menu of contracts he proposes. The standard result of MT 1990 is that the principal strictly benefits from private information with respect to his utility function. MT 1992 show that this result does not carry over to situations where the principal's private information directly enters the agent's pay-off, Chade and Silvers (2001) factor moral hazard with respect to the production stage into these models. They show that there are beliefs supporting a Perfect Bayesian Equilibrium where the principal is better off if his technology becomes less informative. The key difference between the models on informed principals and our paper is that the principal's information is non-verifiable in this literature whereas in our model the contract can be made directly contingent on the principal's verifiable information. Finally, Feess and Walzl (2003) derive the optimal contract in a simple model on delegated expertise, but they do not consider that the principal has additional information.

\section{The model}

A risk-neutral principal has a project requiring initial investment outlays of I. The project is "good" with probability $q_{0}$ and "bad" with probability $1-q_{0}$. If it is good, it yields return $R$. If it is bad, it yields 0 . We assume that the project's expected net return is positive, i.e. $q_{0} R-I>0$.

The principal has two possibilities to improve his knowledge about the project's quality. First, he has costless access to an information technology (IT) that provides a good signal with probability $g$, and a bad signal with probability $1-g$. If the signal is good, the probability for a good project increases to $q_{g}>q_{0}$. If the signal is bad, the probability is reduced to $q_{b}<q_{0}$. Bayesian consistency requires that

$$
g q_{g}+(1-g) q_{b}=q_{0}
$$

Define $q_{i}, i \in\{0, g, b\}$ as the principal's information. We assume that there is no incentive problem associated with the IT, and that the signal is verifiable. Furthermore, we assume that the signal becomes common knowledge in the very moment when the principal checks the IT. ${ }^{1}$

\footnotetext{
${ }^{1}$ Note that this assumption is only used for simplicity. If the principal can check and hide the signal, this leads to a signaling game where the principal may signal his information by his contract. But since the IT is binary in our model, one can easily
} 
Second, the principal can hire a risk-neutral agent who exerts unobservable effort $e$ to detect bad projects. Let $e(\pi)$ be the effort costs required to identify a bad project with probability $\pi$. We assume that $e \in C^{3}$, that $e(0)=0$, $e^{\prime}(0)=0, e^{\prime \prime}(0) \geq 0, e^{\prime \prime \prime}(\pi)>0 \forall \pi \in[0,1)$, and $\lim _{\pi \rightarrow 1} e^{\prime \prime \prime}(\pi)=\infty$.

After having detected a bad project or not, the agent sends message $a_{j}$, $j \in\{d, n\}$, to the principal ( $a_{d}$ for detection, $a_{n}$ for no detection of a bad project). The agent's findings are private information, and the message is not necessarily truthful. The agent's reservation utility is normalized to 0 , and he is protected by limited liability in the sense that his wage must be non-negative in each state of the world. Thus, we consider a principal-agent relationship with hidden action, hidden information and limited liability. The expected utilities of the principal and the agent are denoted $\Phi$ and $U$, respectively.

Since the principal's actions (acquisition of the signal and decision upon investment) are verifiable, any contract specifies the timing of information release and the investment decision contingent on the verifiable information. Therefore there are three classes of contracts or policies. In the first class of contracts, the principal may not engage the agent at all (policy $N$ ) regardless of the signal (as the signal is costless, he will check the IT before he decides upon investment). Second, he can check the IT before he offers a contract to the agent (disclosure policy $D$ ). By definition of policy $D$, the agent is engaged for at least one signal. And third, he can commit to checking the IT after the agent's report (concealment policy $C$ ). Policy $D$ has two advantages. The principal's decision to hire the agent can be based on the signal, and he can adjust his contract offer to the signal. By contrast, the effort choice cannot depend on the signal in policy $C$, since the signal is not known at this time. However, the payments to the agent will be defined contingent on the signal. In stage 0 , the principal chooses between policies $N, D$ and $C$. The extensive forms of the three policies are described in sections 3,4 , and 5 , respectively.

\section{$3 \quad$ No-engagement policy}

If the principal decides not to engage the agent, he will check the IT before he decides upon investment and invests whenever his expected profit is weakly

prove that there is no separating or pooling equilibrium leading to higher profits then the maximum of policy $D$ and $C$. 
positive. Let us define $\phi_{i}^{k}, i \in\{0, g, b\}, k \in\{I, S\}$, as the principal's expected profit if he invests $(I)$ or stops the project $(S)$ with information $q_{i}$ where $\phi_{i}^{S}=0$ and $\phi_{i}^{I}=q_{i} R-I \forall i$. Furthermore, define $\Phi_{N}^{j, h}=g \phi_{g}^{j}+(1-g) \phi_{b}^{h}$, $j, h \in\{I, S\}$ as the principal's expected profit under policy $N$ if he chooses action $j$ for a good signal and action $h$ for a bad signal. Maximum profits under policy $N$ are given by

\section{Proposition 1.}

$$
\Phi_{N}^{\max } \equiv \max _{j, h} \Phi_{N}^{j, h}= \begin{cases}\Phi_{N}^{I, I}, & \text { iff } q_{b} R>I \\ \Phi_{N}^{I, S}, & \text { otherwise }\end{cases}
$$

Proof. As $q_{g} R-I>0$ by assumption, the principal's equilibrium profit is either $\Phi_{N}^{I, S}$ or $\Phi_{N}^{I, I}$. The result follows from $\Phi_{N}^{I, I}-\Phi_{N}^{I, S}=(1-g) \phi_{b}^{I}=$ $(1-g)\left(q_{b} R-I\right)$.

Note that, if $q_{b} R \geq I$, the principal can maximize his profits under policy $N$ simply by not checking the signal at all.

\section{Disclosure policy}

By definition of policy $D$, the agent is engaged for at least one signal. The following Lemma streamlines the analysis.

Lemma 1. (i) If the principal hires the agent, he invests if and only if the agent sends message $a_{n}$. (ii) If the agent chooses $\pi_{i}>0$, he tells the truth.

Proof. Part (i). Suppose not. This means that the principal's decision is independent of the agent's report. But then, hiring the agent has no benefit. Part (ii). If the agent lies, he either sends $a_{d}$ or $a_{n}$ regardless of his findings. Define $\widetilde{U}\left(a_{j}\right)$ as his expected payment in this case. Then, his expected utility is $U\left(a_{j}\right)=\widetilde{U}\left(a_{j}\right)-e\left(\pi_{i}\right)$ which is strictly decreasing in $\pi_{i}$.

Lemma 1 given, the feasible contracts under policy $D$ are depicted in figure 1 .

Insert figure 1 about here

In figure 1, only the subgame in which the signal is checked is explicitly shown since the case where the signal is not checked is isomorphic. Decision nodes are squared, and nature moves are circled. Numbers refer to the stages, "P" to moves by the principal, " $A$ " to moves by the agent and " $N$ " to nature 
moves. When the principal checks the signal in stage 1 , nature decides upon the signal's outcome $q_{i}, i \in\{g, b\}$ in stage 2. For each signal, the principal then chooses his investment strategy $k \in\{I, E, S\}$ in stage 3 where he invests directly for $k=I$, engages the agent for $k=E$, and stops the project for $k=S$. If he engages the agent, the agent's effort choice follows in stage 4 , and the agent detects a bad project with probability $\pi$ in stage 5 . In stage 6 , the agent sends message $a_{d}$ or $a_{n}$, and the principal decides upon investment in stage 7 . Finally, a nature's move determines the outcome in stage 8 .

Analogously to the former section we define $\phi_{i}^{k}(i \in\{0, g, b\}$ and $k \in\{I, E, S\})$ as the principal's profit if the signal is $q_{i}$, and if he invests directly $(k=I)$, engages the agent $(k=E)$, or stops the project $(k=S)$. The principal's profits for a specific contract are given by $\Phi_{D}^{j, h}=g \phi_{g}^{j}+(1-g) \phi_{b}^{h}, j, h \in\{I, E, S\}$ such that the principal chooses action $j$ for signal $q_{g}$ and action $h$ for signal $q_{b} .{ }^{2}$ As the agent is engaged for at least one signal, ${ }^{3}$ and since the principal might not check the IT, we are left with 6 possible types of contracts with expected profits $\Phi_{D}^{I, E}, \Phi_{D}^{E, I}, \Phi_{D}^{E, S}, \Phi_{D}^{S, E}, \Phi_{D}^{E, E}, \phi_{0}^{E}$.

To deduce profits if the agent is engaged and if the principal has information $q_{i}$, let us define the following payments from the principal to the agent: a payment $\tau_{i}$ for message $a_{d}$, a payment $\omega_{i}$ if the investment yields $R$, and a payment $\omega_{i}^{0}$ if the investment fails. If he tells the truth, the agent's utility when deciding upon $\pi_{i}$ is

$$
U_{i}=q_{i} \omega_{i}+\left(1-q_{i}\right)\left(\pi_{i} \tau_{i}+\left(1-\pi_{i}\right) \omega_{i}^{0}\right)-e\left(\pi_{i}\right) .
$$

With probability $q_{i}$, the project is good and yields $R$, so that the agent gets $\omega_{i}$ if he tells the truth. If the project is bad, the agent detects it with probability $\pi_{i}$ and receives $\tau_{i}$. He does not detect it with probability $\left(1-\pi_{i}\right)$, and then he gets $\omega_{i}^{0}$. His effort is thus implicitly given by

$$
\left(1-q_{i}\right)\left(\tau_{i}-\omega_{i}^{0}\right)=e^{\prime}\left(\pi_{i}\right)
$$

where our assumptions on $e\left(\pi_{i}\right)$ ensure an interior solution for the effort if $\tau_{i}-\omega_{i}^{0}>0$. However, the agent also has the opportunity to choose $\pi_{i}=0,{ }^{4}$ and to send either $a_{d}$ or $a_{n}$. In the first case, his utility is $U_{i}\left(a_{d}\right)=\tau_{i}$, while we

\footnotetext{
${ }^{2}$ Recall that we know from Lemma 1 how the principal acts in the remainder of the game for $k=E$.

${ }^{3}$ Otherwise we would be back in policy $N$.

${ }^{4}$ Recall from Lemma 1 that choosing positive effort and lying is dominated by choosing $\pi_{i}=0$ and lying.
} 
have $U_{i}\left(a_{n}\right)=q_{i} \omega_{i}+\left(1-q_{i}\right) \omega_{i}^{0}$ in the second case. Therefore, truth-telling requires that

$$
U_{i} \geq U_{i}\left(a_{d}\right)=\tau_{i}
$$

and

$$
U_{i} \geq U_{i}\left(a_{n}\right)=q_{i} \omega_{i}+\left(1-q_{i}\right) \omega_{i}^{0} .
$$

Note that $U_{i}\left(a_{n}\right)$ is just the special case of $U_{i}$ where $\pi_{i}=0$, so that Ineq. (5) is automatically fulfilled by an interior solution for Eqn. (3). Hence, Ineq. (5) can be neglected in the principal's optimization problem. Furthermore, we have

Lemma 2. $\left(\omega_{i}^{0}\right)^{*}=0 .^{5}$

All remaining proofs are in the Appendix.

Lemma 2 expresses that the agent receives nothing if a project fails. The reason is that the effort decreases in $\omega_{i}^{0}$ (see Eqn. (3) above)), and that $\omega_{i}^{0}$ can be substituted at no cost by $\omega_{i}$ to fulfill the truth-telling constraint (4). In the optimal contract, Ineq. (4) will be binding since $\omega_{i}$ is chosen as low as possible.

We can now formalize the principal's maximization problem if he engages the agent. He maximizes

$$
\phi_{i}^{E}=q_{i}\left(R-I-\omega_{i}\right)-\left(1-q_{i}\right)\left(\pi_{i} \tau_{i}+\left(1-\pi_{i}\right) I\right)
$$

subject to the agent's effort selection given by Eqn. (3), and to the (binding) truth-telling constraint (4) which can be rewritten as

$$
q_{i} \omega_{i}=\tau_{i}\left(1-\pi_{i}+\pi_{i} q_{i}\right)+e\left(\pi_{i}\right) .
$$

Substituting Eqn. (3) and Eqn. (7) into $\phi_{i}^{E}$, and simplifying leads to

$$
\left(\phi_{i}^{E}\right)^{*}=q_{i}(R-I)-\left(1-q_{i}\right)\left(1-\pi_{i}^{*}\right) I-\frac{e^{\prime}\left(\pi_{i}^{*}\right)}{1-q_{i}}-e\left(\pi_{i}^{*}\right)
$$

where the equilibrium effort $e\left(\pi_{i}^{*}\right)$ implemented by the principal is given by ${ }^{6}$

$$
\left(1-q_{i}\right) I=e^{\prime}\left(\pi_{i}^{*}\right)+\frac{e^{\prime \prime}\left(\pi_{i}^{*}\right)}{1-q_{i}},
$$

\footnotetext{
${ }^{5}()^{*}$ indicates optimal values for a certain contract.

${ }^{6}$ Our assumptions on $e(\pi)$ ensure that $\frac{d^{2} \phi_{i}^{E}}{d \pi_{i}^{2}}<0$. If $e^{\prime \prime}(0)>0$, then Eqn.(9) does not necessarily have a solution. Then, however, $\pi_{i}^{*}=0$ such that it is optimal not to engage the agent.
} 
expressing that the marginal savings from detecting bad projects $\left(\left(1-q_{i}\right) I\right)$ must equal the marginal payment to the agent $\left(e^{\prime}\left(\pi_{i}\right)+\frac{e^{\prime \prime}\left(\pi_{i}\right)}{1-q_{i}}\right)$. This marginal payment exceeds the agent's marginal effort costs $e^{\prime}\left(\pi_{i}\right)$ by $\frac{e^{\prime \prime}\left(\pi_{i}\right)}{1-q_{i}}$, so that $\frac{e^{\prime \prime}\left(\pi_{i}\right)}{1-q_{i}}$ can be interpreted as the agent's marginal rent.

For a deeper interpretation of the result, let us define $e\left(\pi_{i}^{f}\right)$ as the effort the principal would implement if there were no incentive problem at all, and $e\left(\widetilde{\pi}_{i}^{*}\right)$ as the effort if the agent's findings were observable. ${ }^{7}$ Then we get

Proposition 2. For all $i \in\{g, b, 0\}$, we have (i) $\pi_{i}^{*}<\tilde{\pi}_{i}^{*}<\pi_{i}^{f}$. (ii) $\left(\phi_{i}^{E}\right)^{*}<\left(\widetilde{\phi}_{i}^{E}\right)^{*}<\phi_{i}^{f}$. (iii) $\frac{d\left(\phi_{i}^{f}\right)^{*}}{d q_{i}}>0$, (iv) $\frac{d\left(\widetilde{\phi}_{i}^{E}\right)^{*}}{d q_{i}}>0, \quad(v) \frac{d\left(\phi_{i}^{E}\right)^{*}}{d q_{i}} \lesseqgtr 0$. (vi) $\left(\Phi_{D}^{E, E}\right)^{*} \lesseqgtr\left(\phi_{0}^{E}\right)^{*}$.

Part (i) and (ii) express that, for all $q_{i}$, the agent's equilibrium effort and the principal's profit are highest without incentive problem, followed by the situation with moral hazard only. Hence, the agent's private information aggravates the incentive problem via Eqn. (7), which leads to lower effort and lower profits. Parts (iii) and (iv) express that the principal strictly benefits from a higher probability of a project beeing good if the agent has no private information.

However, this is not necessarily the case with a truth-telling problem (Part $(\mathrm{v}))$. This surprising result is due to the following countervailing effect: suppose the principal wants to implement some probability $\hat{\pi}_{i}$. Recall that $\hat{\pi}_{i}$ is determined by $e^{\prime}\left(\hat{\pi}_{i}\right)=\left(1-q_{i}\right) \tau_{i}$. It follows that the detection premium $\hat{\tau}_{i}$ required to implement probability $\hat{\pi}_{i}$ strictly increases in $q_{i}$ (since the agent knows that he will be paid less often, he demands a higher premium). But the utility the agent can get when he sends message $a_{d}$ without working at all is simply $\tau_{i}$, and therefore independent of $q_{i}$. Hence, for any effort the principal wishes to implement, the right-hand side of the truth-telling constraint (7) increases in $q_{i}$, which means that the principal must also offer a higher expected utility if he wants to ensure that the agent tells the truth. If this rent effect dominates, the principal's profit decreases in $q_{i}$. Part (vi) is a direct consequence of the rent effect and says that, even though the IT is costless and certainly informative, the principal may be better off by not checking the signal before the effort decision is made by the agent. Of course, this means that the principal's profit is concave in $q_{i}$.

\footnotetext{
${ }^{7} \phi_{i}^{f}, \widetilde{\phi}_{i}^{E}$, and $\left(\widetilde{\phi}_{i}^{E}\right)^{*}$ are defined accordingly.
} 
This given, we can now analyze optimality of the specific contracts under policy $D$.

Proposition 3. $\Phi_{D}^{\max } \equiv \max _{j, h} \Phi_{D}^{j, h} \in\left\{\left(\Phi_{D}^{E, E}\right)^{*},\left(\Phi_{D}^{E, S}\right)^{*},\left(\Phi_{D}^{I, E}\right)^{*}\right\}$.

Obviously, $\left(\Phi_{D}^{S, E}\right)^{*}$ and $\left(\Phi_{D}^{E, I}\right)^{*}$ are dominated. If $q_{g} \rightarrow 1$, it is optimal to invest directly so that $\left(\Phi_{D}^{I, E}\right)^{*}$ is maximum. ${ }^{8}$ For $q_{b} \rightarrow 0$, the project will be stopped and $\left(\Phi_{D}^{E, S}\right)^{*}$ is maximum. Furthermore, the agent will be engaged whenever $e^{\prime \prime}(0)$ is sufficiently small and $q_{i} \in(0,1)$. This given, either $\left(\phi_{0}^{E}\right)^{*}$ or $\left(\Phi_{D}^{E, E}\right)^{*}$ may be maximum due to the counter-intuitive effects caused by truth-telling (see Proposition 2).

\section{Concealment policy}

By definition of policy $C$, the signal is checked after the agent's report. In contrast to policy $D$ there is no sub-case without hiring the agent, since we would otherwise be back in policy $N$. Furthermore, part (ii) of Lemma (1) is still valid and implies that the agent reports truthfully in equilibrium such that the principal will stop the project after receiving message $a_{d}$. This given, there are two possible contracts: the principal can always invest after $a_{n}$, or he can only invest if the agent sends message $a_{n}$ and the signal is good. In the first case, profits are denoted as $\Phi_{C}^{R}$, while we use $\Phi_{C}^{S}$ in the latter case ${ }^{9}$. The extensive form of policy $C$ is shown in figure 2 . The key difference to figure 1 is that the principal offers a contract at stage 1 and checks the signal before he decides upon investment in stage 5 .

Insert figure 2 here.

To derive $\Phi_{C}^{R}$, let us define $\tau_{i}$ with $i \in\{G, B\}$ (as the detection premium if the signal is good $(i=G)$ or bad $(i=B)$. Analogously, define $\omega_{G}$ and $\omega_{B}$ as the wage if $R$ is realized when the signal is good or bad. Lemma 2 carries over to policy $C$ as there is no benefit of paying the agent if a project fails. Thus, the principal's profit is

$$
\begin{aligned}
\Phi_{C}^{R}= & g\left[q_{g}\left(R-\omega_{G}-I\right)-\left(1-q_{g}\right)\left(1-\pi_{R}\right) I-\left(1-q_{g}\right) \pi_{R} \tau_{G}\right] \\
& +(1-g)\left[q_{b}\left(R-\omega_{B}-I\right)-\left(1-q_{b}\right)\left(1-\pi_{R}\right) I-\left(1-q_{b}\right) \pi_{R} \tau_{B}\right] .
\end{aligned}
$$

\footnotetext{
${ }^{8}$ Recall that $\Phi_{D}^{I, S}$ and $\Phi_{D}^{I, I}$ is excluded by the definition of policy $D$.

${ }^{9} \pi_{R}$ and $\pi_{S}$ are defined accordingly where superscripts " $R$ " and " $S$ " indicate that the principal relies on the agent's report or on the signal, respectively.
} 
The agent's utility if he chooses effort $e\left(\pi_{R}\right)$ and reports truthfully is

$$
U_{R}=g\left[q_{g} \omega_{G}+\left(1-q_{g}\right) \pi_{R} \tau_{G}\right]+(1-g)\left[q_{b} \omega_{B}+\left(1-q_{b}\right) \pi_{R} \tau_{B}\right]-e\left(\pi_{R}\right) .
$$

Thus, the agent's effort if he tells the truth is implicitly given by

$$
g\left(1-q_{g}\right) \tau_{G}+(1-g)\left(1-q_{b}\right) \tau_{B}=e^{\prime}\left(\pi_{R}\right) .
$$

If the agent sends message $a_{d}$ without working at all, his utility is $(1-g) \tau_{B}+$ $g \tau_{G}$, so that truth-telling requires ${ }^{10}$

$$
U_{R} \geq(1-g) \tau_{B}+g \tau_{G} .
$$

Hence, the principal's profit as given by Eqn. (10) must be maximized subject to the agent's effort selection constraint (12) and the binding truth-telling constraint (13).

The following Lemma is crucial for understanding policy $C$.

Lemma 3. (i) A detection premium will only be paid if the signal is bad. (ii) Whether the wage for successful projects is contingent on the signal or not is irrelevant.

The advantage of policy $C$ is that the truth-telling problem can be mitigated by paying a detection premium only if the signal is bad. The reason is that the signal provides a stochastic update on the honesty of the agent's report so that the expected wage if he sends $a_{n}$ can be reduced. Hence, inducing one unit of effort is cheaper if the premium is only paid if the agent's report matches the signal. Conversely (see Lemma 3(ii)), making $\omega_{i}(i=G, B)$ contingent on the signal has no impact, because the agent will never report $a_{n}$ without working at all. In the following, we assume without loss of generality that $\omega_{B}=0$.

Taking Lemma 3 into account, substituting Eqn. (12) and Eqn. (13) into the principal's profit function (10), making use of $g q_{g}+(1-g) q_{b}=q_{0}$, and simplifying yields equilibrium profits

$$
\left(\Phi_{C}^{R}\right)^{*}=q_{0}(R-I)-\left(1-\pi_{R}^{*}\right)\left(1-q_{0}\right) I-\frac{e^{\prime}\left(\pi_{R}^{*}\right)}{1-q_{b}}-e\left(\pi_{R}^{*}\right)
$$

where the agent's equilibrium effort $e\left(\pi_{R}^{*}\right)$ solves

\footnotetext{
${ }^{10}$ Again, the second truth-telling constraint is fullfilled due to the effort selection constraint.
} 


$$
\left(1-q_{0}\right) I=\frac{e_{R}^{\prime \prime}\left(\pi_{R}^{*}\right)}{1-q_{b}}+e^{\prime}\left(\pi_{R}^{*}\right) .
$$

$\left(\Phi_{C}^{S}\right)^{*}$ is derived analogously in the Appendix, and the agent's equilibrium effort implemented by the principal is implicitly given by ${ }^{11}$

$$
g\left(1-q_{g}\right) I=\frac{e^{\prime \prime}\left(\pi_{S}^{*}\right)}{1-q_{b}}+e^{\prime}\left(\pi_{S}^{*}\right) .
$$

The main insights on policy $C$ are summarized in

Proposition 4. (i) $\frac{d e\left(\pi_{R}^{*}\right)}{d q_{g}}=0$, (ii) $\frac{d\left(\Phi_{C}^{R}\right)^{*}}{d q_{g}}=0$, (iii) $\frac{d\left(\Phi_{C}^{R}\right)^{*}}{d q_{b}}<0$, (iv) $\pi_{g}^{*}<$ $\pi_{R}^{*}<\pi_{b}^{*}$, (v) $\pi_{S}^{*}<\pi_{R}^{*}$, (vi) $\left(\Phi_{C}^{R}\right)^{*} \gtreqless\left(\Phi_{C}^{S}\right)^{*}$.

Parts (i) and (ii) express that both the effort and the profit are independent of the quality of the good signal if the principal always invests after message $a_{n}$. As any change in $q_{g}$ leads to a change in $g$ when the other parameters remain unchanged, the principal's profit function has to be independent of both $q_{g}$ and $g$ which can be seen from Eqn. (14). For an intuition, first recall from the agent's effort selection constraint that the marginal expected payment required to implement a certain effort given by $\tau_{B}(1-g)\left(1-q_{b}\right)$ is independent of $g$, because it does not make any difference whether the same expected premium is realized with high $g$ and high $\tau_{B}$ or with low $g$ and low $\tau_{B}$. Furthermore, the information rent the principal must offer to fulfill the truth-telling constraint is also independent of $q_{g}$ and $g$, since the utility the agent gets if he sends message $a_{d}$ without working at all is $\tau_{B}(1-g)=\frac{\bar{e}_{R}^{\prime}}{1-q_{b}}$. Part (iii) follows from the fact that the opportunity to reduce rents decreases in $q_{b}$ which leads to lower profits. That $e\left(\pi_{R}^{*}\right)$ is in-between the efforts implemented in policy $D$ for good and bad signals is intuitive, since exerting effort is more valuable if the number of bad projects is high (part(iv)). $e\left(\pi_{S}^{*}\right)<e\left(\pi_{R}^{*}\right)$ is due to the fact that detecting bad projects is less important for $\left(\Phi_{C}^{S}\right)^{*}$, since the principal will never invest if the signal is bad anyway (part $(\mathrm{v})$ ). Finally, $\left(\Phi_{C}^{R}\right)^{*}>\left(\Phi_{C}^{S}\right)^{*}$ if the bad signal is not too informative and $R$ is sufficiently large; $\left(\Phi_{C}^{R}\right)^{*}=\left(\Phi_{C}^{S}\right)^{*}$ if $g \rightarrow 1$ (i.e. if the good signal becomes uninformative); otherwise it is better to invest only with message

\footnotetext{
${ }^{11}$ As in the former section our assumptions on $e(\pi)$ ensure convexity of the principal's profit function. If $e^{\prime \prime}(0)>0$, Eqn.(15) and (16) do not necessarily have a solution. Then, however, $\pi_{i}^{*}=0(i \in\{R, S\})$ such that we are back in policy $N$.
} 
$a_{n}$ and a good signal (part (vi)). Since the equilibrium efforts for the two alternatives are different, no simple threshold values for $q_{b}$ can be defined. Analogously to policy $D$, we define $\Phi_{C}^{\max }$ as the maximum profit reachable under policy $C$.

\section{Comparison of the three policies}

First of all, we analyze when it is superior not to engage the agent at all.

Proposition 5. Sufficient conditions for Policy $N$ being optimal are (i) $\frac{e^{\prime \prime}(0)}{\left(1-q_{b}\right)}>\left(1-q_{b}\right) I$ and $($ ii $) q_{g}=1$ and $q_{b}=0$.

Intuitively, part (i) refers to the case where it is too costly to implement any positive effort. Part (ii) is straightforward as the agent can not provide useful information if the signal is perfect anyway. For the following comparison of policy $C$ and $D$, it is assumed that policy $N$ is inferior ${ }^{12}$.

We start with the cases where the specific advantages of policy $C$ and $D$ disappear, so that clear results can be established.

Proposition 6. (i) Suppose there is no truth-telling problem. Then, policy $D$ is superior.

(ii) If the principal prefers not to check the IT under policy D, then policy $C$ is superior.

Since the only advantage of policy $C$ compared to policy $D$ is that the truthtelling problem can be mitigated, $C$ must be inferior without truth-telling problem. For part (ii), recall from Proposition 2 that it may be optimal under policy $D$ to engage the agent without checking the IT (i.e. $\left.\Phi_{D}^{\max }=\left(\phi_{0}^{E}\right)^{*}\right)$. This implies that the principal does not want to implement different efforts, so the signal is worthless in $D$. But with policy $C$, the principal can still reduce the agent's rent, so that policy $D$ is inferior and not using the IT can never be optimal.

Next, we turn to the case where the agent is engaged for both signals under policy $D$ (which yields profits $\left.\left(\Phi_{D}^{E, E}\right)^{*}\right)$. All partial derivatives of $\Delta \equiv \Phi_{C}^{\max }-$ $\Phi_{D}^{\max }$ are ambiguous in sign. For instance, $\partial \Delta / \partial q_{g}$ and $\partial \Delta / \partial q_{b}$ both vary in sign, depending on the explicit form of $e(\pi)$, so that there is no generally valid connection between the IT's informativeness and the ranking of the policies. However, there is a clear result as to the superiority of policy $C$ :

\footnotetext{
${ }^{12} \mathrm{~A}$ sufficient condition for policy $N$ being inferior is $e^{\prime \prime}(0)=0$ together with $q_{i} \in(0,1)$.
} 
Proposition 7. Suppose $\Phi_{D}^{\max }=\left(\Phi_{D}^{E, E}\right)^{*}$. Then there is an $\epsilon>0$ such that policy $C$ is optimal as long as $q_{g} \in\left(q_{0}, q_{0}+\epsilon\right)$.

First note that $\Phi_{C}^{R} \rightarrow \Phi_{C}^{S}$ if $q_{g} \rightarrow q$ (and $g \rightarrow 1$ ), since the two contracts under policy $C$ are identical if the probability for a bad signal converges to zero. Hence, Proposition 7 holds for $\Phi_{C}^{R}$ and for $\Phi_{C}^{S}$. Proposition 7 then says that policy $C$ is superior when the good signal is sufficiently uninformative. For an intuition, first recall from Proposition 4 that the equilibrium profit in policy $C$ is independent of $q_{g}$. In policy $D$, however, the good signal is of little value if $q^{g} \rightarrow q$. Furthermore, since $(1-g) \rightarrow 0$ for $q_{g} \rightarrow q$, profits when the signal is bad are almost negligible. Hence, uninformative ITs strongly support policy $C$.

Conversely, there are no comparably simple constellations where policy $D$ is superior. To show that $\Delta>0$, we only had to consider situations where the benefit of implementing different efforts under policy $D$ (almost) disappears. These kinds of constellations do not exist for $\Delta<0$, because the IT can always be exploited to reduce the rent in policy $C$. Hence, the comparison depends on the shape of the $e(\pi)$-function and on all other variables such that we are restricted to instructive examples that illustrate the superiority of policy $D$.

Proposition 8. (i) There is a set of parameter values $\left(R, I, q_{0}\right)$ and a cost function $e(\pi)$ such that policy $D$ is superior if $\left(q_{g}-q_{b}\right)$ is sufficiently large. (ii) Suppose $\Phi_{D}^{j, h}=\Phi_{D}^{\max }$, then there exists a set of parameter values and a cost function such that $\Phi_{C}^{\max }>\Phi_{D}^{\max }$ for all $(j, h)$.

For part (i), note that a highly informative IT works in favor of policy $D$ since the contract can be adjusted to the different signals. Furthermore, we know from Proposition 7 that an informative IT is required to generate $\Delta<0$. However, there are no values of $q_{g}$ and $q_{b}$ leading to $\Delta<0$ regardless of all other parameters for two reasons: first, if the IT becomes too informative, it is better not to engage the agent at all, so that policy $N$ is optimal. Second, if the agent is not engaged for one signal under policy $D$, the reduction in the information rent may dominate. This holds for all feasible contracts under policy $D$ (part (ii)). 


\section{Discussion}

We have analyzed optimal contracts in a principal-agent model with hidden action, limited liability and truth-telling constraints under the assumption that the principal has access to additional verifiable information. The main question was whether the principal should commit himself to check the IT before (policy $D$ ) or after (policy $C$ ) the agent's effort decision. We have shown that policy $D$ is superior if there is no truth-telling problem, so that the parameter constellations do not matter in the pure moral hazard setting. However, as soon as truth-telling becomes a problem, we found strong support for concealment (policy $C$ ) as the optimal timing. First, policy $C$ is better if the signal is sufficiently uninformative (i.e. if the IT is almost worthless as an update of the project's characteristics, but can still be exploited as a statistics of the agent's behavior). Second, the principal's profit in policy $D$ can be decreasing in the percentage of good projects $\left(q_{i}\right)$, because higher $q_{i}$ 's aggravate the truth-telling problem. Then, policy $C$ is superior as it makes (efficient) use of the (costless) IT while the principal may prefer not to check the technology under policy $D$. Third, we have shown that the rentreduction effect of policy $C$ is quite robust with respect to the project and IT characteristics such that concealment should be considered as a valuable option in reality.

If a scoring system for a bank loan is known to be not very informative for the case at hand, it is indeed optimal for the bank to run the IT after the loan officer has submitted his report. In cases without a pronounced truthtelling problem (like journal-refereeing), however, an immediate disclosure of existing information proves useful. Most problems of delegated expertise (like investigations by doctors or lawyers etc.) lie between these benchmark cases and the commitment of the principal to remain uninformed should at least be considered as a worthy alternative.

Let us now discuss robustness in light of our assumptions. In one of our motivating examples with a loan officer, risk-aversion seems to be a natural extension. Under policy $C$, the detection premium would still be higher for a bad signal, but the principal would also pay positive wages if the signal and the report do not coincide to improve risk-sharing. Hence, risk aversion reduces the benefits from policy $C$. On the other hand, depending on the quality of $q_{b}$ and $q_{g}$, the wages for the two subgames under policy $D$ may be quite different, and this is a drawback of policy $D$ with risk-aversion. The trade-off analyzed in the paper would still exist with risk-aversion, but the 
analysis would become less transparent.

The assumption that the agent has zero wealth is strong. Instead, one could assume that the agent has some wealth $W>0$, but not enough to buy the project. Then, the principal can reduce the agent's rent under policy $D$ by charging the agent's wealth as an up-front payment. Under policy $C$, the principal would punish the agent if the signal and the agent's report differ. Again, the model becomes less clear, but the trade-off would persist.

Our assumption that the agent detects bad projects with some probability can be interpreted in the sense that the project is certainly bad if the agent gets a bad signal. On the other hand, if he does not detect a bad project, the probability for a good project is not equal to one. Hence, one might think about removing this asymmetry by introducing a positive probability for a good project if the agent gets a bad signal. However, this results in a more cumbersome analysis without further insights.

Also, one might question that the signal is costlessly available - running a computer-based scoring system or sending a public agent may not come at no cost. However, the comparison between the three policies could then hardly be handled in this general form, and the surprising result that it may be superior under policy $D$ not to check the IT even without costs would be less clear.

Finally, the principal-agent relationship considered in our paper may be a repeated one. In an (infinitely) repeated game the following should be an equilibrium: with some (arbitrarily small) probability the principal invests even if the agent reports $a_{d}$, and threatens to end the contractual relationship if the project yields $R$ (which means that the agent has lied with probability one). This should almost eliminate the truth-telling problem, since the agent loses all of his future rents. However, investing even with signal $a_{d}$ may not be renegotiation-proof, so that additional questions arise that are beyond the objective of our model. 


\section{Appendix A: Proofs}

Proof of Lemma 2 For any effort $\hat{\pi}_{i}>0$ the principal wants to implement, he has to fulfill the agent's truth-telling constraint

$$
q_{i} \omega_{i}+\left(1-q_{i}\right)\left(\hat{\pi}_{i} \tau_{i}+\left(1-\hat{\pi}_{i}\right) \omega_{i}^{0}\right)-e\left(\hat{\pi}_{i}\right) \geq \tau_{i}
$$

and the effort selection constraint

$$
\left(1-q_{i}\right)\left(\tau_{i}-\omega_{i}^{0}\right)=e^{\prime}\left(\hat{\pi}_{i}\right)
$$

$\omega_{i}$ is chosen such that Eqn.(17) is binding because $\omega_{i}$ has no impact on the agent's effort. Substituting the binding constraints into the principal's profit function

$$
\phi_{i}^{E}=q_{i}\left(R-I-\omega_{i}\right)-\left(1-q_{i}\right)\left(\hat{\pi}_{i} \tau_{i}+\left(1-\hat{\pi}_{i}\right)\left(I+\omega_{i}^{0}\right)\right)
$$

yields after simplifying

$$
\phi_{i}^{E}=q_{i} R-I+\left(1-q_{i}\right) \hat{\pi}_{i} I-\frac{e^{\prime}\left(\hat{\pi}_{i}\right)}{1-q_{i}}-e\left(\hat{\pi}_{i}\right)-\omega_{i}^{0}
$$

and hence

$$
\frac{d \phi_{i}^{E}}{d \omega_{i}^{0}}=-1<0
$$

Since this holds for any $e\left(\hat{\pi}_{i}\right)$ the principal wants to implement, it must also hold for the optimal effort.

Proof of Proposition 2 Part (i). The first-best effort $e\left(\pi_{i}^{f}\right)$ is implicitly given by $\left(1-q_{i}\right) I=e^{\prime}\left(\pi_{i}^{f}\right)$. For the principal's profit in the pure moral hazard case $\left(\widetilde{\phi}_{i}^{E}\right)$, we insert Eqn.(3) and $\omega_{i}=0$ into Eqn.(6). This yields

$$
\left(\widetilde{\phi}_{i}^{E}\right)^{*}=q_{i}(R-I)-\left(1-q_{i}\right)\left(1-\widetilde{\pi}_{i}^{*}\right) I-\widetilde{\pi}_{i}^{*}\left(e^{\prime}\left(\widetilde{\pi}_{i}^{*}\right)\right)
$$

with the optimal effort $e\left(\tilde{\pi}_{i}^{*}\right)$ implicitly given by

$$
\left(1-q_{i}\right) I=e^{\prime}\left(\widetilde{\pi}_{i}^{*}\right)+\widetilde{\pi}_{i}^{*} e^{\prime \prime}\left(\widetilde{\pi}_{i}^{*}\right) .
$$

From $\frac{1}{1-q_{i}}>1>\tilde{\pi}_{i}$, it follows that $\pi_{i}^{*}<\tilde{\pi}_{i}^{*}<\pi^{f}$. Part(ii). To prove $\left(\widetilde{\phi}_{i}^{E}\right)^{*}-\left(\phi_{i}^{E}\right)^{*}>0$, suppose the principal implements $\pi_{i}^{*}$ even without a 
truth-telling problem ${ }^{13}$, which does not maximize his profits. But even then, $\widetilde{\phi}_{i}^{E}\left(\pi_{i}^{*}\right)-\left(\phi_{i}^{E}\right)^{*}>0$ as $\pi_{i}<\frac{1}{1-q_{i}}$. Hence, $\left(\widetilde{\phi}_{i}^{E}\right)^{*}>\widetilde{\phi}_{i}^{E}\left(\pi_{i}^{*}\right)>\left(\phi_{i}^{E}\right)^{*}$ where the first inequality follows from the definition of optimality. $\phi_{i}^{f}>\left(\widetilde{\phi}_{i}^{E}\right)^{*}$ can be proven analogously ${ }^{14}$. Part(iii). $\frac{d\left(\phi_{i}^{f}\right)^{*}}{d q_{i}}=R>0 . \quad \operatorname{Part}(i v) . \quad \frac{d\left(\widetilde{\phi}_{i}^{E}\right)^{*}}{d q_{i}}=$ $R-\tilde{\pi}_{i}^{*} I>0$. Part $(v)$. From $\frac{d\left(\phi_{i}^{E}\right)^{*}}{d q_{i}}=R-\pi_{i}^{*} I-\frac{e^{\prime}\left(\pi_{i}^{*}\right)}{\left(1-q_{i}\right)^{2}}$, it follows that profits are monotonically increasing in $q_{i}$ if $R$ is sufficiently large, but can be monotonically decreasing for low values of $R$ as shown in Example 2 in Appendix B. Part(vi). In Example 3(2) in Appendix B we provide a specification of $e(\pi)$ that implies that $\left(\phi_{i}^{E}\right)^{*}$ is concave (convex) in $q_{i}$ such that $\left(\Phi_{D}^{E, E}\right)^{*}<(>)\left(\phi_{0}^{E}\right)^{*}$.

Proof of Proposition 3 Since $q_{0} R-I>0$ by assumption, and since $q_{g}>q_{0}$, we have $\phi_{i}^{I}>\phi_{i}^{S}$, for $i \in\{0, g\}$ which excludes $\left(\Phi_{D}^{S, E}\right)^{*}$ as maximum. Optimality of $\left(\Phi_{D}^{E, I}\right)^{*}$ requires that $\phi_{g}^{I}<\phi_{g}^{E}$ and $\phi_{b}^{I}>\phi_{b}^{E}$ hold simultaneously. But this is impossible due to $\frac{\left(d \phi_{i}^{I}\right)^{*}}{d q_{i}}=R>\frac{\left(d \phi_{i}^{E}\right)^{*}}{d q_{i}}=R-\pi_{i}^{*} I-\frac{e\left(\pi_{i}^{*}\right)}{\left(1-q_{i}\right)^{2}}$. To see that each of the remaining contracts can be optimal, note that a sufficient condition to engage the agent is $e^{\prime \prime}(0)=0$ together with $q_{i} \in(0,1)$. On the other hand, sufficient conditions not to engage the agent are $e^{\prime \prime}(0) \geq$ $\left(1-q_{i}\right)^{2} I, q_{b}=0$ or $q_{g}=1$. Hence,

- $\left(\Phi_{D}^{E, S}\right)^{*}$ is maximum if e.g. $e^{\prime \prime}(0)=0, q_{b}=0$, and $q_{g}<1$.

- $\left(\Phi_{D}^{I, E}\right)^{*}$ is maximum if e.g. $e^{\prime \prime}(0)=0, q_{b}>0$, and $q_{g}=1$.

That $\left(\phi_{0}^{E}\right)^{*}$ and $\left(\Phi_{D}^{E, E}\right)^{*}$ can be maximum is proven by Examples 2 and 3, respectively.

Proof of Lemma 3 We prove the Lemma only for $\Phi_{C}^{R}$. The proof for $\Phi_{C}^{S}$ proceeds analogously and is available on request.

\footnotetext{
${ }^{13}$ Note that $U_{i}^{\prime \prime}<0 \forall \pi_{i}>0$ and $U_{i}=0$ if $\pi_{i}=0$ such that the agent's effort selection constraint Eqn. (3) guarentees $U_{i}>0$ for all $\pi_{i} \in\left(0, \tilde{\pi}_{i}^{*}\right]$. This implies that the agent's participation constraint is fulfilled.

${ }^{14}$ Proof available on request.
} 
The principal's profit $\Phi_{C}^{R}$ as given in the main body of the text must be maximized subject to the effort-selection-(ESC) and the truth-telling constraints (TTC) (see Eqn. (12) and Eqn. (13) in the text). Optimization with respect to $\omega_{G}$ and $\omega_{B}$ yields

$$
\begin{aligned}
\frac{\partial L}{\partial \omega_{B}} & =-(1-g) q_{b}+\lambda_{T T C}(1-g) q_{b}, \\
\frac{\partial L}{\partial \omega_{G}} & =-g q_{g}+\lambda_{T T C} g q_{g},
\end{aligned}
$$

such that $\lambda_{T T C}=1$ (i.e. a binding TTC) solves the necessary conditions simultaneously. Thus, all combinations of $\omega_{G}$ and $\omega_{B}$ that satisfy the TTC are optimal. As to $\tau_{G}$ and $\tau_{B}$, the set of first-order conditions is given by $\lambda_{T T C}=1$ and

$$
\begin{aligned}
\frac{\partial L}{\partial \tau_{G}} & =\lambda_{E S C} g\left(1-q_{g}\right)-g=0 \\
\frac{\partial L}{\partial \tau_{B}} & =\lambda_{E S C}(1-g)\left(1-q_{b}\right)-(1-g)=0 .
\end{aligned}
$$

This system of equations cannot be solved simultaneously. Isolating $\lambda_{E S C}$ in the first equation yields $\lambda_{E S C}=\frac{1}{\left(1-q_{g}\right)}$, implying that $\frac{\partial L}{\partial \tau_{B}}>0$. This clearly establishes a minimum. With the second equation, we get $\lambda_{E S C}=\frac{1}{\left(1-q_{b}\right)}$ such that $\frac{\partial L}{\partial \tau_{G}}<0$. Therefore, the optimal payment scheme is given by $\tau_{G}=0$ and any $\tau_{B}, \omega_{G}, \omega_{B}$ that satisfy ESC and TTC.

Proof of Proposition 4 Part (i) and (ii). Since $q_{g}$ neither directly nor indirectly (via $g$ ) enters into $\Phi_{C}^{R}$, it follows that $\frac{d e\left(\pi_{R}^{*}\right)}{d q_{g}}=0$ and $\frac{d\left(\Phi_{C}^{R}\right)^{*}}{d q_{g}}=0$. $\operatorname{Part}(i i i)$. $\frac{d\left(\Phi_{C}^{R}\right)^{*}}{d q_{b}}=-\frac{e^{\prime}\left(\pi_{R}^{*}\right)}{\left(1-q_{b}\right)^{2}}<0$. Part (iv). A comparison of the FOCs Eqn. (9) and (15) yields $e\left(\pi_{g}^{*}\right)<e\left(\pi_{R}^{*}\right)<e\left(\pi_{b}^{*}\right)$.

Part (v). If the principal invests only with message $a_{n}$ and with a good signal, he maximizes

$$
\Phi_{C}^{S}=g\left[q_{g}\left(R-\omega_{G}-I\right)-\left(1-q_{g}\right)\left(1-\pi_{S}\right) I\right]-(1-g)\left(1-q_{b}\right) \pi_{S} \tau_{B}
$$

subject to the same constraints (Eqn. (12) and Ineq. (13)). Using $g q_{g}+$ $(1-g) q_{b}=q_{0}$, substituting into $\Phi_{C}^{S}$ and simplifying yields

$$
\left(\Phi_{C}^{S}\right)^{*}=g q_{g} R-g I+g\left(1-q_{g}\right) \pi_{S}^{*} I-\frac{e^{\prime}\left(\pi_{S}^{*}\right)}{1-q_{b}}-e\left(\pi_{S}^{*}\right)
$$


with $\pi_{S}^{*}$ given by

$$
g\left(1-q_{g}\right) I=\frac{e^{\prime \prime}\left(\pi_{S}^{*}\right)}{1-q_{b}}+e^{\prime}\left(\pi_{S}^{*}\right)
$$

such that $e\left(\pi_{R}^{*}\right)>e\left(\pi_{S}^{*}\right)$.

Part(vi) Define $T \equiv\left(\Phi_{C}^{R}\right)^{*}-\left(\Phi_{C}^{S}\right)^{*}$ to get

$$
\begin{aligned}
T= & R\left(q_{0}-g q_{g}\right)-I\left(1-g-\left(1-q_{0}\right) \pi_{R}^{*}+g\left(1-q_{g}\right) \pi_{S}^{*}\right) \\
& -\frac{e^{\prime}\left(\pi_{R}^{*}\right)-e^{\prime}\left(\pi_{S}^{*}\right)}{1-q_{b}}-\left(e\left(\pi_{R}^{*}\right)-e\left(\pi_{S}^{*}\right)\right)
\end{aligned}
$$

where the first term is positive and the last two terms are negative due to $\pi_{S}^{*}<\pi_{R}^{*}$. Since $q_{0}>g q_{g}$, a sufficient condition for $T>0$ is that $R$ is large. On the other hand, if $q_{b} \rightarrow 0$ this implies $g q_{g} \rightarrow q_{0}$ such that $T \rightarrow-I\left(1-g-\left(1-q_{0}\right) \pi_{R}^{*}+g\left(1-q_{g}\right) \pi_{S}^{*}\right)-e^{\prime}\left(\pi_{R}^{*}\right)+e^{\prime}\left(\pi_{S}^{*}\right)-e\left(\pi_{R}^{*}\right)-e\left(\pi_{S}^{*}\right)$ as $q_{b} \rightarrow 0$. Even if the principal suboptimally implements $\pi_{R}^{*}$ under policy $S$, we have $T \rightarrow-I(1-g)\left(1-\pi_{R}^{*}\right)<0$ as $q_{b} \rightarrow 0$. Hence, $T<0$ for the optimal $\pi_{S}^{*}$. Finally, note that as $g \rightarrow 1$ (and consequently $q_{g} \rightarrow q_{0}$ ) $\pi_{S}^{*} \rightarrow \pi_{R}^{*}$ and therefore $T \rightarrow 0$.

Proof of Proposition 5 Part(i). If $e^{\prime \prime}(0)>\left(1-q_{b}\right)^{2} I$ none of the first order conditions Eqs. (9), (15), and (16) can be fulfilled such that it is always superior not to engage the agent. Part(ii). Obvious.

Proof of Proposition 6 Part(i). From Eqn.(22) in the proof of Proposition 2, we know that the principal's profit without a signal and without truth-telling problem is

$$
\widetilde{\phi}_{0}^{E}=q_{0}(R-I)-\left(1-q_{0}\right)\left(1-\widetilde{\pi}_{0}\right) I-\widetilde{\pi}_{0} e^{\prime}\left(\widetilde{\pi}_{0}\right) .
$$

To get profits under policy $C$ without truth-telling problem, we insert $\omega_{G}=$ $\omega_{B}=0$ and Eqn. (12) into the respective profit functions Eqn. (14) and (25). This yields

$$
\begin{aligned}
& \widetilde{\Phi}_{C}^{R}=q_{0}(R-I)-\left(1-q_{0}\right)\left(1-\widetilde{\pi}_{R}\right) I-\widetilde{\pi}_{R} e^{\prime}\left(\widetilde{\pi}_{R}\right) . \\
& \widetilde{\Phi}_{C}^{S}=g\left(q_{g} R-I\right)-g\left(1-q_{g}\right)\left(1-\widetilde{\pi}_{S}\right) I-\widetilde{\pi}_{S} e^{\prime}\left(\widetilde{\pi}_{S}\right) .
\end{aligned}
$$

First note that $\left(\widetilde{\Phi}_{C}^{R}\right)^{*}=\left(\widetilde{\phi}_{0}^{E}\right)^{*}$ since profits are of the same functional form and first order conditions are therefore identical. Both profits are dominated 
by $\left(\widetilde{\Phi}_{D}^{E, E}\right)^{*}=g\left(\widetilde{\phi}_{g}^{E}\right)^{*}+(1-g)\left(\widetilde{\phi}_{b}^{E}\right)^{*}$, i.e. the profits in policy $D$ without truthtelling problem if the agent is engaged for both signals: as $\widetilde{\phi}_{i}^{E}$ is convex in $q_{i}\left(\frac{d^{2}\left(\widetilde{\phi}_{i}^{E}\right)^{*}}{d q_{i}^{2}}=-\frac{d \widetilde{\pi}_{i}^{*}}{d q_{i}} I>0\right)$, we get $\left(\widetilde{\Phi}_{D}^{E, E}\right)^{*}>\left(\widetilde{\phi}_{0}^{E}\right)^{*}=\left(\widetilde{\Phi}_{C}^{R}\right)^{*}$. As to $\widetilde{\Phi}_{C}^{S}$, note that $g \cdot\left(\widetilde{\phi}_{g}^{E}\right)^{*}>\left(\widetilde{\Phi}_{C}^{S}\right)^{* 15}$.

Part(ii).

$$
\begin{aligned}
\left(\Phi_{C}^{R}\right)^{*}-\left(\phi_{0}^{E}\right)^{*} & =\left(1-q_{0}\right)\left(\pi_{R}^{*}-\pi_{0}^{*}\right) I-\frac{e^{\prime}\left(\pi_{R}^{*}\right)}{1-q_{b}}+\frac{e^{\prime}\left(\pi_{0}^{*}\right)}{1-q_{0}}-e\left(\pi_{R}^{*}\right)+e\left(\pi_{0}^{*}\right) \\
& >e^{\prime}\left(\pi_{0}^{*}\right) \frac{q_{0}-q_{b}}{\left(1-q_{0}\right)\left(1-q_{b}\right)} \geq 0
\end{aligned}
$$

where the first inequality is due to a suboptimal implementation of $\pi_{0}^{*}$ in policy $C^{16}$.

Proof of Proposition 7 First, recall from the proof of Proposition 4 that $\Phi_{C}^{S} \rightarrow \Phi_{C}^{R}$ for $g \rightarrow 1$. Therefore, it needs to be proven that, for any arbitrarily chosen $q_{b}, \Delta>0$ for $q_{g} \rightarrow q_{0}$.

Step 1. Consider policy $C$, and suppose the principal implements the same effort as without an additional signal, that is $\pi_{R}=\pi_{0}^{*}$. Even then, $\Phi_{C}^{R}\left(\pi_{0}^{*}\right)>$ $\left(\phi_{0}^{E}\right)^{*}$ as $\frac{e^{\prime}\left(\pi_{0}^{*}\right)}{1-q^{b}}-\frac{e^{\prime}\left(\pi_{0}^{*}\right)}{1-q}<0$. By definition of optimality, $\left(\Phi_{C}^{R}\right)^{*}=\Phi_{C}^{R}\left(\pi_{R}^{*}\right)>$ $\Phi_{C}^{R}\left(\pi_{0}^{*}\right)$, hence $\left(\Phi_{C}^{R}\right)^{*}>\left(\phi_{0}^{E}\right)^{*}$.

Step 2. For policy $D$, recall that the fraction of profits that can be influenced by the principal's decision is

$$
g\left[\left(1-q_{g}\right) \pi_{g}^{*} I-\frac{e^{\prime}\left(\pi_{g}^{*}\right)}{1-q_{g}}-e\left(\pi_{g}^{*}\right)\right]+(1-g)\left[\left(1-q_{b}\right) \pi_{b}^{*} I-\frac{e^{\prime}\left(\pi_{b}^{*}\right)}{1-q_{b}}-e\left(\pi_{b}^{*}\right)\right] .
$$

For any given $q_{b}, \lim _{q_{g} \rightarrow q_{0}}(1-g)\left[\left(1-q_{b}\right) \pi_{b}^{*} I-\frac{e^{\prime}\left(\pi_{b}^{*}\right)}{1-q_{b}}-e\left(\pi_{b}^{*}\right)\right]=0$. Moreover, $\lim _{q_{g} \rightarrow q_{0}} g\left[\left(1-q_{g}\right) \pi_{g}^{*} I-\frac{e^{\prime}\left(\pi_{g}^{*}\right)}{1-q_{g}}-e\left(\pi_{g}^{*}\right)\right]=\left(1-q_{0}\right) \pi_{0}^{*} I-\frac{e^{\prime}\left(\pi_{0}^{*}\right)}{1-q_{0}}-e\left(\pi_{0}^{*}\right)$. Thus, the principal's profit under policy $D$ converges towards $\left(\phi_{0}^{E}\right)^{*}$. Hence, $\lim _{q_{g} \rightarrow q_{0}} \Delta>0$.

\footnotetext{
${ }^{15}$ The proof proceeds analogously to the proof of Proposition 2(ii) and is available on request.

${ }^{16}$ Recall from Example 1 and 3 that $\Phi_{D}^{\max }=\left(\phi_{0}^{*}\right)^{*}$ is indeed feasible.
} 
Proof of Proposition 8 Part(i). Proven by the existence of Example 4 in Appendix B. Part(ii). We have to give an example for every feasible contract with profit $\left(\Phi_{D}^{i, j}\right)^{*}$ under policy $D$ that is not ruled out by Proposition 3 such that $\Phi_{D}^{\max }=\left(\Phi_{D}^{i, j}\right)^{*}$ and $\Phi_{C}^{\max }>\left(\Phi_{D}^{i, j}\right)^{*}$. First, according to Proposition 6(ii) $\left(\phi_{0}^{E}\right)^{*}$ is always dominated by policy $C$. Second, according to Proposition 7, $\left(\Phi_{D}^{E, E}\right)^{*}$ can be dominated by $\left(\Phi_{C}^{R}\right)^{*}$ and $\left(\Phi_{C}^{S}\right)^{*}$. We are thus left with $\left(\Phi_{D}^{I, E}\right)^{*}$ and $\left(\Phi_{D}^{E, S}\right)^{*}$. Examples 5,6 prove the existence of parameter sets that support each of these contracts as being optimal under policy $D$, and show that they can be dominated by policy $C$. 


\section{Appendix B: Examples}

Example 1: Assume that $e(\pi)=\left(\frac{\pi}{a}\right)^{N}$. The first order condition given by Eqn. (9), can be abbreviated by

$$
A_{i}=B_{i} e^{\prime \prime}\left(\pi_{i}\right)+e^{\prime}\left(\pi_{i}\right)
$$

(with $A_{i}>0, B_{i}>0$ ). Then Eqn. (9) becomes

$$
A_{i}=\frac{B_{i} N(N-1)}{a^{2}}\left(\frac{\pi_{i}}{a}\right)^{N-2}+\frac{N}{a}\left(\frac{\pi_{i}}{a}\right)^{N-1}
$$

such that $\pi_{i}^{*}$ must solve

$$
\pi_{i}^{*}=\left(A_{i} /\left(\frac{N}{a^{2}}\left(B_{i}(N-1)+\pi_{i}^{*}\right)\right)\right)^{1 /(N-2)} \cdot a .
$$

For $N \rightarrow \infty$ the right-hand side of Eqn. (32) converges towards $a^{17}$ independent of $A_{i}$ and $B_{i}$ such that $\pi_{i}^{*} \uparrow a \forall i \in\{0, g, b\}$. From this it follows that $\Phi_{D}^{\max }=\left(\phi_{0}^{E}\right)^{*}$.

Example 2: Assume that $e(\pi)=C I \frac{1}{2} \pi^{2}+\epsilon(-\ln (1-\pi)-\pi)$. As long as $\epsilon>0$, this effort function satisfies all our standard requirements, while for $\epsilon$ sufficiently small and $\pi_{i} \in[0,1)$, the optimization program can be restricted to $e\left(\pi_{i}\right)=C I \frac{1}{2} \pi_{i}^{2}$. Therefore, the FOC is approximated by

$$
\begin{aligned}
\left(1-q_{i}\right) I & =\frac{e^{\prime \prime}\left(\pi_{i}\right)}{1-q_{i}}+e^{\prime}\left(\pi_{i}\right) \\
& =\frac{C I}{1-q_{i}}+C I \pi_{i}
\end{aligned}
$$

such that

$$
\pi_{i}^{*}=\frac{1-q_{i}}{C}-\frac{1}{1-q_{i}} .
$$

Recall that $\pi \in[0,1)$ is a necessary condition for approximating $e(\pi)=$ $C I \frac{1}{2} \pi^{2}$. This requires $C \in\left(C_{0}, C_{1}\right]$ with $C_{0}=\frac{\left(1-q_{i}\right)^{2}}{2-q_{i}}$ and $C_{1}=\left(1-q_{i}\right)^{2}$. We use this specification of $e(\pi)$ to give an example for a (locally) monotone decreasing profit function. In

$$
\frac{d\left(\phi_{i}^{E}\right)^{*}}{d q_{i}}=R-\pi_{i}^{*} I-\frac{e^{\prime}\left(\pi_{i}^{*}\right)}{\left(1-q_{i}\right)^{2}}
$$

\footnotetext{
${ }^{17}$ As $\lim _{N \rightarrow \infty}(A N)^{-B / N}=1 \forall A, B \in R$.
} 
we restrict ourselves to the minimum revenue $R=\frac{I}{q_{0}}$. Inserting the optimal effort we get

$$
\begin{aligned}
\frac{d\left(\phi_{i}^{E}\right)^{*}}{d q_{i}} & =I\left(\frac{1}{q_{0}}-\pi_{i}^{*}-\frac{C \pi_{i}^{*}}{\left(1-q_{i}\right)^{2}}\right) \\
& =I\left(\frac{1}{q_{0}}-\frac{1-q_{i}}{C}+\frac{C}{\left(1-q_{i}\right)^{3}}\right) .
\end{aligned}
$$

Solving $\frac{d \phi_{i}^{E}}{d q_{i}}=0$ yields $C_{c r i t}=\frac{1}{2 q_{0}}\left(\left(1-q_{i}\right)^{2}\left(q_{i}-1+\sqrt{\left(1-q_{i}\right)^{2}+4 q_{0}^{2}}\right)\right)$. As $\frac{d^{2} \phi_{i}^{E}}{d q_{i} d C}>0, \frac{d \phi_{i}^{E}}{d q_{i}}<0$ if $C<C_{c r i t}$. This is only feasible if $C_{c r i t} \in\left(C_{0}, C_{1}\right]$. Since $C_{0}=C_{\text {crit }}$ if $q_{i}=\frac{2-3 q_{0}}{1-q_{0}}$ and $C_{1}=C_{k r i t}$ if $q_{i}=1$, it follows there is always a $C<C_{\text {crit }}$ that guarantees $\frac{d\left(\phi_{i}^{E}\right)^{*}}{d q_{i}}<0$ if $q_{0} \in\left[\frac{1}{2}, \frac{2}{3}\right]$ and $q_{i} \in\left(\frac{2-3 q}{1-q}, 1\right)$.

Furthermore, for $e(\pi)=C I \frac{1}{2} \pi^{2}$ the principal's profit is convex in $q_{i} \in\left(q_{b}, q_{g}\right)$, as it can be shown that $\frac{d^{2} \phi_{i}^{E}}{d q_{i}^{2}}=\frac{I}{C}+\frac{3 C}{\left(1-q_{i}\right)^{4}}>0$ such that $\left(\Phi_{D}^{E, E}\right)^{*}>\left(\phi_{0}^{E}\right)^{*}$.

Example 3: Assume $e(\pi)=\pi^{3}+\pi$ and $R=10, I=3$.

Result: If $q_{i} \in\left[0.3, \frac{2}{3}\right] \frac{\partial^{2}\left(\phi_{i}^{E}\right)^{*}}{\partial q_{i}^{2}}<018$

\footnotetext{
${ }^{18}$ Note that this profit is indeed feasible, as $q_{0} R>I$ and $\pi_{i}^{*} \in(0,1)$ in this case (as $e^{\prime}(0)=1, \pi_{i}^{*}>0$ requires that $\left(1-q_{i}\right) I>1$ and hence $\left.q_{i}<2 / 3\right)$. Detailed calculation available on request.
} 
For all the following examples ${ }^{19}$, we use the effort function

$$
e(\pi)=\frac{1}{1-\pi}-1
$$

Example 4: $R=1.000 .000, I=10.000, q_{0}=0.42, q_{g}=0.97$.

Result: If $q_{b} \in[0.228,0.42]$ then $\Phi_{D}^{\max }=\Phi_{D}^{E, E}>\Phi_{C}^{R}=\Phi_{C}^{\max }$.

Example 5: $\quad R=1.000 .000, I=10.000, q_{0}=0.8, q_{g}=0.98$.

Result: If $q_{b} \in[0.1,0.67]$ then $\Phi_{D}^{\max }=\Phi_{D}^{I, E}<\Phi_{C}^{R}=\Phi_{C}^{\max }$.

Example 6: $\quad R=1.000 .000, I=10.000, q_{0}=0.3, q_{b}=0.0001$.

Result: If $q_{g} \in[0.3,0.9]$ then $\Phi_{D}^{\max }=\Phi_{D}^{E, S}<\Phi_{C}^{S}=\Phi_{C}^{\max }$.

\footnotetext{
${ }^{19}$ Detailed calculations available on request.
} 


\section{References}

[1] Beaudry, P. (1994): Why an Informed Principal May Leave Rents to an Agent, International Economic Review, 35, 4, 821-832.

[2] Cella, M. (2001): Informed Principals with Correlation, London School of Economics, working paper.

[3] Chade, H. and Silvers, R. (2001): Informed Principals, Moral Hazard, and the Value of a More Informative Technology, Arizona State University, working paper.

[4] Feess, E. and Walzl, M. (2003): Delegated expertise - when are good projects bad news?, Economics Letters, forthcoming.

[5] Demski, J. and D. Sappington (1987a): Delegated Expertise, Journal of Accounting Research, 25 , 68-89.

[6] Demski, J. and D. Sappington (1987b): On the Timing of Information Release, Information Economics and Policy, 2, 307-316.

[7] Lewis, T.R. and D. Sappington (1997): Information Management in Incentive Problems, Journal of Political Economy, 105, 4, 796-821.

[8] Maskin, E. and J. Tirole (1990): The Principal-Agent Relationship with an Informed Principal: The Case of Private Values, Econometrica, 58, 379-409.

[9] Maskin, E. and J. Tirole (1992): The Principal-Agent Relationship with an Informed Principal, II: Common Values, Econometrica, 60, 1-42. 